\title{
ARTERIAL EMBOLISM IN THE LIMBS \\ Clinical Assessment and Management
}

\author{
By A. L. JACobs, M.A., D.M., M.R.C.P. \\ Physician to the Whittington Hospital
}

\section{Material}

The views expressed in this paper are based on a study of clinical and morbid anatomical material, personally observed by the writer during the period 1938 to 1954 . It comprises:

(I) Clinical records of a series of 122 separate incidents of limb embolism in 69 patients.

(2) Records of 48 autopsies on patients of the clinical series.

(3) An additional series of 269 patients suffering from mitral stenosis, examined for evidence of old arterial occlusions in the limbs.

(4) An anatomical study of arterial collaterals, made on 66 human subjects by post-mortem arteriography.

(A fuller account of this study is to be published.)

\section{Spontaneous Recovery in Limb Embolism}

Examination of the limb pulses and enquiry into the history of patients with mitral stenosis show that many of them have had embolism of the limbs with unassisted recovery. In the patients with auricular fibrillation, the proportion showing this was found to be 27.3 per cent. and to increase with increasing age. In patients with regular heart rhythm the corresponding figure was 4 . I per cent.

\section{Aetiology}

Only a single embolic incident in the series of 122 occurred in a patient who had no heart disease. Auricular fibrillation was present in 91 incidents, combined with mitral stenosis in 64. Four incidents occurred in patients with mitral stenosis and regular heart rhythm. Bacterial endocarditis accounted for $I_{3}$ incidents and myocardial infarction for nine. In $3 \mathrm{I}$ incidents congestive heart failure was present at the time of the embolism but the incidence of associated non-cardiac disease was relatively low. Embolism occurred much more frequently while the patient was at rest than during activity. Thirteen embolic incidents occurred in close relation to over-dosage of digitalis (in two of these the heart rhythm was regular).

\section{Consecutive Thrombosis}

Local thrombosis in an artery after occlusion by an embolus (" consecutive ' or 'secondary' thrombosis) has been regarded by most writers as an $\underset{\perp}{\omega}$ important adverse factor which may precipitate $\omega_{0}$ gangrene. Post-mortem dissection of the oc- or cluded artery in 64 embolized limbs did not bear के out this view. It was found that gangrene had not occurred more frequently in limbs with major $\frac{5}{3}$ degrees of consecutive thrombosis than in those $\vec{\phi}$ with minor degrees. Extensive thrombosis oc- $\mathbb{D}$ curred in some limbs without provoking gangrene $\frac{\mathbb{D}}{\mathbb{D}}$ and massive gangrene occurred in some limbs with 3 little or no thrombosis. It appeared that the extent of the consecutive thrombosis was detero $\overrightarrow{0}$ mined mainly by the anatomical course of the collateral circulation.

\section{Symptoms}

The onset of symptoms (hereafter referred to as ' onset') was sudden in 79 per cent. of incidents but there were some with gradual and undramatic onset. The commonest local symptom was pain, present in 7I per cent.; it was usually sited well distal to the position of the embolus and only in I 5 per cent. was there any record of pain in the region of the occlusion. The only other common local symptoms, in order of frequency, were subjective coldness, numbness, loss of muscular power and tingling of the extremity ("pins and needles').

In a small number of cases of embolism of large arteries (aortic and iliac) there were general symptoms superficially resembling those of surgical $\frac{D}{O}$ shock but differing from this in that the bloodpressure tended to be raised above normal level in $N$ the initial period.

\section{Signs}

Suppression of all arterial pulses distal to the occlusion was by no means invariable. In embolism of the aortic bifurcation or the iliac $\mathbb{D}$ artery, it was not uncommon during the initial period for a pulse to be palpable, though usually 

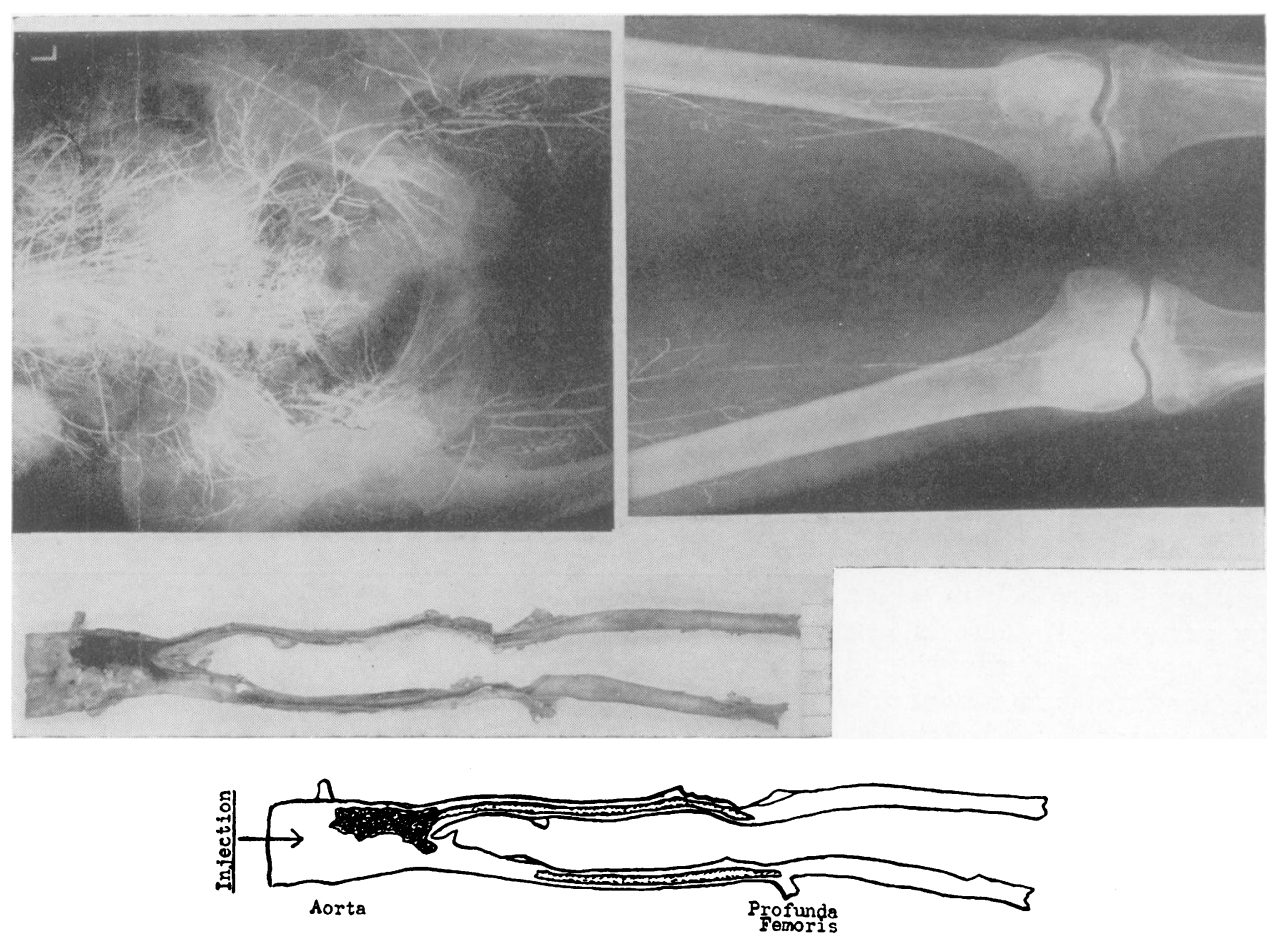

FIG. I.-A case of embolism of both lower limbs. Post-mortem arteriography and autopsy specimen. Post-mortem arteriography demonstrates filling of the right common iliac artery, which the autopsy specimen shows to be the only patent part of the two ilio-common femoral trunks. There is partial filling of the two superficial femoral arteries by collateral flow but the injection has not developed sufficient pressure in these vessels to carry the fluid beyond the knees. In this case, both lower limbs survived without gangrene; the autopsy was made nearly two years later, when the patient died of subarachnoid haemorrhage.

weakened, for some distance beyond the embolus. In such cases the more distal pulses in the limb were usually suppressed.

Colour changes in the skin of the limb were common: pallor in severe ischaemia and cyanosis in less severe ischaemia. Lowering of skin temperature was usually similar in extent to colour changes. The ischaemic skin signs were always limited to an area well distal to the site of the embolus. Demonstrable muscular weakness, loss of tendon reflexes and sensory impairment were noted in the more severe cases. Signs of ischaemic muscle damage (swelling and tenderness, especially in the calf) were seen in about 20 per cent. of incidents, though subsequent fibrotic contracture was uncommon.

Extravasation staining (distinguished from cyanosis by failure to blanch on pressure) usually heralded massive gangrene; it never appeared less than 18 hours after onset and rarely less than $3^{6}$ hours.

\section{Embolic Arteriospasm}

Many writers on embolism hold the view that the impaction of an embolus often sets up a condition of spasm in the occluded artery and that this may extend into the collateral arteries, thus aggravating the ischaemia. In the experience of the writer, embolic arteriospasm is a rare phenomenon and there was no evidence that it can extend into collateral arteries. Moreover, critical examination of the evidence allows the conclusion that the observations on which the theory has been based can mostly be explained without postulating arteriospasm. The importance of this factor in the genesis of ischaemia appears to have been greatly exaggerated.

\section{Diagnosis}

Embolism of a limb artery produces an acute ischaemic syndrome clinically indistinguishable from that due to any other form of acute arterial occlusion. The ischaemic symptoms may be severe or mild and their onset may be sudden or gradual. Recognition of an arterial occlusion as embolic depends, not on any special distinguishing features in the ischaemic syndrome itself, but on associated evidence, i.e. the presence of heart dis- 
ease of ' emboligenic' type (auricular fibrillation, rheumatic valvular disease, bacterial endocarditis or myocardial infarction). The great majority of cases occur in patients with mitral stenosis and auricular fibrillation.

\section{Differential Diagnosis}

There are relatively few forms of acute arterial occlusion which may simulate embolism and for purposes of clinical diagnosis they may be reduced to the following:

(I) Local arterial thrombosis.

(2) Acute arterial spasm: (a) in thrombophlebitis; (b) 'pseudo-embolism.'

Local Arterial Thrombosis. This is usually due to arteriosclerotic intimal disease but evidence of this does not exclude embolism, which often occurs in elderly persons. If there is heart disease of appropriate type the occlusion should be regarded as embolic, for there is no way of proving that it is not, and treatment must be guided by this assumption. Conversely, a diagnosis of embolism is rarely justified in the absence of heart disease.

Thrombo-phlebitic Arteriospasm. In occasional cases of thrombo-phlebitis affecting a deep venous trunk, the adjacent arterial trunk may go into spasm, possibly through irritation of its adventitia by the inflammatory process. This will produce an acute ischaemic syndrome, usually rather mild, though the pulses may be temporarily suppressed. In one such case of the writer's, there were two small pulmonary emboli and these, together with the absence of heart disease, made the diagnosis clear.

'Pseudo-embolism.' Many observers have described cases of transient acute ischaemic syndrome, closely resembling embolism, in which it was subsequently proved that there was no occluding embolus. Strangely enough, these cases occur almost exclusively in patients who have ' $\mathrm{em}$ boligenic' heart disease and this makes diagnosis very difficult. The causation of 'pseudo-embolism' is obscure but it seems to be due to arterial spasm, which may last for some days. By analogy with well-recognized types of arterial spasm, especially traumatic spasm, it must be suspected that this condition results from some lesion of the arterial wall and Leriche suggested that it might be due to ' micro-embolism' or haemorrhage affecting one of the vasa vasorum.

Pseudo-embolism is almost impossible to distinguish clinically from embolic occlusion but it is relatively rare and does not often cause serious diagnostic error. The series of 122 embolic incidents included four examples.

\section{Localization of an Embolus}

The main difficulty in determining the site of an cmbolus arises from the fact, already mentioned? that in a considerable number of cases of embolisn of the larger arteries (mainly aortic and iliac) the pulses are not immediately suppressed beyond the. embolus but may remain palpable (though weakened), sometimes for 24 hours or more ('Nordentoft's sign'). Despite this fact, pulsê deficiencies are the only reliable evidence on which to assess the site of an embolus. The essentiab principle to bear in mind is that a weakened pulse has the same significance for localization as ane absent pulse. This principle will prove mis $\overrightarrow{0}$ leading only in the event of a complicating arteriatspasm, extending proximally along the arteria trunk from the embolus and in the writer's experience this is a very rare phenomenon.

We may therefore say that an embolus almostw. invariably lies between the most distal unimpaire ${ }_{\omega}^{\perp}$ pulse and the most proximal impaired pulse. Infe conjunction with the fact that it usually lies at as point of main bifurcation, this enables the majority of emboli to be accurately localized. 의

(In the writer's experience, 'Aleman's sign,' i.e. tenderness over the artery at the site of the embolus, is a relatively late and inconstant sign, of little use in diagnosis.)

Because of persistent pulsation beyond the sited of an embolus, the distinction between embolisinnof the aortic bifurcation and simultaneous separateer embolism of the two lower limbs can sometimes $\overline{\mathrm{B}} \mathrm{e}^{\infty}$ difficult but the principles already emphasizedo should usually enable a decision to be made. If doubt remains, it is better to regard the embolismo as aortic, for this is rather more common and itso urgency is greater.

Some of the diagnostic difficulties might be $\overrightarrow{\vec{O}}$ resolved by arteriography but this can rarely be 3 carried out quickly enough.

\section{'Relapsing Ischaemic Syndrome'}

Many cases of embolism, even some in large arteries such as the iliac, develop adequate col- 3 . lateral circulation and recover spontaneously, often without a diagnosis having been made. In some of these cases, perhaps months later, there may be $\circ$ an acute general deterioration of the circulation, usually because of heart failure, with consequento sharp fall of blood-pressure. When this occurs, the collateral circulation, previously adequate, $\sigma$ may suddenly fail and an acute ischaemic syndrome $N$ will result which can easily be mistaken for a new స్ embolic occlusion. Several such cases were seen and in one of them the error led to the performance of a futile operation. Regular recording of limb pulses as a routine in all cases of heart disease when $\stackrel{\odot}{?}$ they first come under observation will sometimes prevent an error of this kind. 


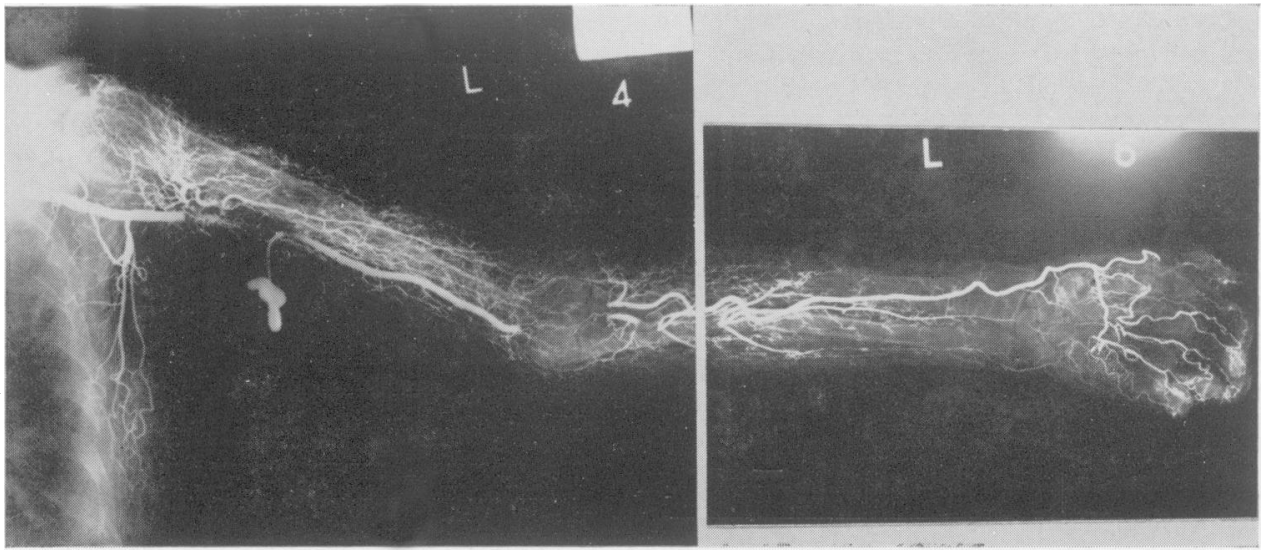

FIG. 2.-Experimental post-mortem arteriography. Demonstration of collateral circulation in an upper limb. The brachial artery has been occluded at two levels: (I) in its highest portion and (2) at its division into radial and ulnar arteries. In each case multiple ligatures have been applied so as to exclude a segment of about $5 \mathrm{~cm}$. in length from the circulation. The injection has been made distally into the brachial trunk immediately below the upper occlusion, at a pressure of $235 \mathrm{~mm}$. mercury. The occluded segments are shown in the arteriogram as gaps in the arterial trunk and at both points an excellent collateral circulation has been demonstrated.

\section{Outcome}

A large number of cases of limb embolism recover spontaneously without coming under medical observation and cases seen in hospital therefore represent only a proportion of the whole -mainly those with the more severe ischaemic symptoms. The overall recovery rate is consequently considerably higher than that observed in hospital series.

Because of the heart disease and its associated hazards, the general mortality in limb embolism is much greater than could be accounted for by the effects of the limb embolism alone. We are here concerned with the local result in the limb, assessed as far as possible independently of the general result in the patient. (When the patient dies of some other cause before the local result in the limb can be fairly assessed, the case is classified as ' indeterminate.')

As a basis of comparison for different groups of limb embolisms, the " limb survival rate ' ('L.S.R.') can be used; this is the proportion in which the limb survives without gangrene, expressed as a percentage of all incidents in the group (including indeterminate incidents).

The results in the whole clinical series, including both treated and untreated incidents, are shown in Table $\mathbf{~}$.

It will be seen that the L.S.R. varies inversely with the size of the occluded artery. In brachial and popliteal embolism, gangrene is rare; in fact no instance was seen in 14 cases of brachial embolism (the L.S.R. is not 100 per cent. because
TABLE I

Limb Survival Rates in in6 Incidents of Limb EMBOLISM

\begin{tabular}{|c|c|c|}
\hline \multicolumn{2}{|c|}{ Site of embolism } & Limb survival rate \\
\hline \multirow{2}{*}{ Upper limb arteries } & Axillary & 75 per cent. \\
\hline & Brachial & 86 per cent. \\
\hline \multirow{4}{*}{ Lower limb arteries } & Aorta & 23 per cent. \\
\hline & Iliac & 48 per cent. \\
\hline & Femoral & 74 per cent. \\
\hline & Popliteal & 89 per cent. \\
\hline
\end{tabular}

there were two indeterminate cases). There were two instances of gangrene in 26 cases of popliteal embolism but in both the patient was moribund from other causes when gangrene developed.

\section{The Influence of Treatment on Outcome}

Medical Treatment. The chief methods of medical treatment used were anticoagulant drugs and methods to promote vaso-dilatation: i.e. drugs (papaverine or benzyl-imidazoline hydrochloride), warming the torso with an electric cradle or local injection of the regional sympathetic ganglia. The L.S.R. has been estimated in $4 \mathrm{I}$ embolic incidents so treated and in 68 untreated incidents and the results are shown in Table 2 .

Table 2 shows that the L.S.R. in medically treated embolic incidents, even those in which 
TABLE 2

The Effect of Medical Treatment on Limb Survival Rate IN Limb EMBolism

\begin{tabular}{c|c|c}
\hline Groups of cases & \multicolumn{2}{|c}{ Limb survival rate } \\
\cline { 2 - 3 } Embolism at all sites & Treated cases & Untreated cases \\
\hline $\begin{array}{c}\text { Aortic, iliac and fe- } \\
\text { moral embolism }\end{array}$ & 46 per cent. & 57 per cent. \\
\hline $\begin{array}{l}\text { Aortic, iliac and fe- } \\
\text { moral embolism, all } \\
\text { treated within } \\
\text { hours of onset of } \\
\text { symptoms }\end{array}$ & 63 per cent. & \\
\hline
\end{tabular}

treatment was begun within ro hours of the onset, is not significantly higher than that in untreated incidents.

\section{Surgical Treatment}

Only six of the writer's cases were treated by embolectomy, with survival of the limb in three cases. For evidence on the results of embolectomy we must turn to the larger published series. In eight of the best known collected series from I928 to 1948 , varying in numbers from 18 cases to $\mathbf{2 8 2}$ cases, there is only one series (the smallest) in which a L.S.R. of more than 50 per cent. is claimed for lower limb embolism. In the writer's untreated cases the corresponding figure is $7 \mathrm{I}$ per cent. No doubt surgical series contain proportionately more examples of the grave ischaemic syndromes than an untreated series and this probably explains their less favourable results but it certainly cannot be shown statistically that embolectomy increases the chance of survival of the limb.

It would not be right to conclude that embolectomy never saves a limb but these figures indicate that the number of cases in which this is achieved is small-too small to have any statistical effect on the results of a large series. Some factor other than treatment must play a much greater role in determining outcome. In the writer's view this factor is the natural anatomical endowment of arterial collaterals. It is only in persons who have inadequate collaterals that embolectomy could improve the chance of survival of the limb and if this anatomical deficiency is present only in a small minority then the number of true operative successes must be too small significantly to affect the results in a large series.

This conclusion is supported by the anatomical study of arterial collaterals by post-mortem arteriography, in which it was found that there is a proportion of persons with deficient arterial collaterals. In general, the incidence of deficiency in collaterals for the arteries studied (femoral, popliteal, upper brachial and lower brachial) was found to correspond quite well with the clinical incidence of gangrene in embolism at the corresponding anatomical sites.

\section{Prognosis}

If it be true that embolectomy can be of value only in a limited number of cases of limb embolism, then it becomes important to define the indications for operation and to establish the clinical data which enable these indications to be recognized with the minimum of delay. This is the problem in clinical prognosis.

It has been shown that the outlook for an embolized limb depends very much upon the site $\underset{\perp}{\omega}$ of the arterial occlusion. In embolism of the $\omega$ brachial and popliteal arteries, unless the patient $f$ be moribund, the risk of gangrene is negligible and there is therefore rarely any clinical problem of $\$$

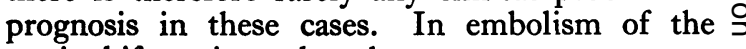
aortic bifurcation, though spontaneous recovery $\vec{C}$ does occur in some cases, the prospect is usually very poor. Moreover, aortic embolism is peculiar in that many cases which initially appear to be improving suddenly regress and develop gangrene and in the early stages there is no means of reo cognizing cases which will deteriorate in this way For these reasons, there is no clinical problem of prognosis in aortic embolism: the outlook should always be assumed to be bad. The main problem is that of diagnosis, already discussed.

It is in embolism of the iliac, femoral and occasionally the axillary arteries that the problem of clinical prognosis is most acute. We have to recognize as early as possible those cases in which gangrene threatens.

\section{Tempo of Improvement in Recovering Limbs}

Correlation of the early ischaemic signs with the subsequent fate of the limb has shown that in a recovering limb obvious improvement of the circulation should appear within two hours of onset. If signs of grave ischaemia (see below) are still present after this, then natural recovery is highly unlikely. The signs which proved most useful in assessing the gravity of the ischaemia were:

(I) Pallor of the whole foot or hand.

(2) Sensory impairment of the whole foot or hand.

(3) Emptiness of the superficial veins of the foot or hand, or very sluggish refilling from the periphery after expression.

If these signs are still present two hours after onset they indicate inadequacy of arterial collaterals and probable gangrene. 


\section{Clinical Management and Treatment}

Some writers have advocated a preliminary period of medical treatment for up to three hours, operation to be undertaken if there is no improvement. The writer's experience does not justify this policy; it indicates that a decision whether to operate should and usually can be made without this undesirable delay.

\section{Embolectomy}

Though the number of cases in which gangrene can be averted by embolectomy is limited, this operation holds first place in the treatment of embolism of the larger limb arteries. Most surgeons are agreed that there is little prospect of success in cases operated on more than ten hours after onset; indeed, the margin may well be less than this, perhaps five to six hours. Assuming that the case is seen early enough, the indications for embolectomy can be very briefly stated:

(I) All cases of aortic embolism.

(2) Cases of embolism of the iliac, femoral or axillary arteries in which signs of grave ischaemia are still present two hours after onset.

Embolectomy is not usually indicated for brachial or popliteal embolism.

\section{Medical Treatment}

Anticoagulant Drugs. Consecutive thrombosis seems to be of less importance in determining the fate of an embolized limb than has been supposed. If this be true, anticoagulant drugs can have little effect on the local result in the limb. However, there are other reasons for using them if no special contraindication exists. Any patient who has had one embolism is very liable to have others and the evidence indicates that this is usually due to the formation of new thrombi in the heart. With the aim of preventing this, anticoagulant drugs should usually be given for a period of at least two to three weeks, irrespective of the condition of the embolized limb. Phenindione does not seem to be harmful to ischaemic tissues as dicoumarol may sometimes be.

Vaso-dilatation. In the writer's experience, vaso-dilator drugs have proved of little use in the treatment of embolism. Apart from this, there are theoretical reasons against their use, for they act generally and therefore must, if effective at all, tend to cause a fall of blood-pressure. There are good grounds for believing that this may impair collateral circulation in the embolized limb. The same consideration applies to the use of a warming cradle over the torso. The only vasodilator method free from this objection is injection of the regional sympathetic ganglia and this is therefore the method of choice, though in the writer's limited experience its effect is not impressive.

General Management. An ischaemic limb should be kept at rest, not restrained and protected from pressure. It should neither be warmed nor actively chilled and is best kept exposed at ordinary room temperature. It must not be elevated and there may be some value in keeping it below body level. The most important element of general care is treatment of the heart disease to maintain the general circulation at its best and avoid a fall of blood-pressure. Overdosage of digitalis must be avoided and diuretics used with discretion in order not to produce undue haemo-concentration, which may precipitate intracardiac thrombosis and so increase the risk of further embolism.

\section{Amputation}

Once gangrene is evident, there is nothing to be gained by delaying amputation. On the contrary, the rapid deterioration often seen in a patient with a gangrenous limb may sometimes be arrested by prompt amputation. This is especially necessary if, as is often the case, there is another embolized limb in which collateral circulation might be endangered by a serious decline in the patient's general condition.

\section{BIBLIOGRAPHY}

ALLEN, E. V., BARKER, N. W., and HINES, E. A., Jr. (1955),

'Peripheral Vascular 'Diseases,' 2nd ed., Philadelphia.

ANDRUS, W. de W. (1950), Arch. Surg. (Chicago), 60, 51 I.

ATLAS, L. N. (1942), Surg. Gynec. Obstet., 74, 236.

BURT, C. C., LEARMONTH, J. R., and RICHARDS, R. L. (1952), Edinb. med. F., N.S.59, 65.

DANZIS, M. (1933), Ann. Surg., 98, 249 and 422.

FLASHER, J., and STEPHENSON, W. (1952), Angiology, 3, 249. GESENIUS, H., and NEUBART, P. (1950), Berliner med. $Z$., $\mathrm{I}, 400$.

HAIMOVICI, H. (1937), ' Les Embolies Artérielles des Membres,' Paris.

HAIMOVICI, H. (1950), Angiology, I, 20.

HOLDEN, W. D. (1952), 'Acute peripheral arterial embolism,' Springfield, Ill.

JEPSON, R. P. (1955), Brit. med. f., ii, 405.

KEY, E. (1936), Brit. F. Surg., 24, 350.

LERICHE, R. (1947), 'Les Embolies de l'Artère Pulmonaire et des Artères des Membres,' Paris.

LEWIS, T. (1936), Clin. Sci., 2, 237.

LUND, C. C. (1937), Ann. Surg., 106, 880.

McCLURE, R. D., and HARKINS, H. N. (1943), Surgery, 14, 747.

MILWIDSKY, H. (1947), Acta med. orient., 6, 213.

MILWIDSKY, H., EHRENFELD, E. N., and de VRIES, A. (1952), Angiology, 3, 275 .

OLOVSON, T. (194I), Acta chir. scand., 86, Sup. 67.

PEARSE, H. E., Jr. (1933), Ann. Surg., 98, 17.

PETITPIERRE, M. (1928), Schweiz. med. Wschr., 28, 700.

REYNOLDS, J. T., and JIRKA, F. J. (1944), Surgery, 16, 485.

RICHARDS, R. L. (1954), Quart. F. Med., N.S.23, 73.

STROMBECK, J. P. (1935), Acta chir. scand., 77, 229.

VEAL, J. R., and DUGAN, T. J. (1951), Ann. Surg., 133, 603. 